\title{
BCH codes for 5G wireless communication systems over multipath fading channel
}

\author{
Ghasan Ali Hussain ${ }^{1}$, Lukman Audah ${ }^{2}$ \\ ${ }^{1}$ Department of Electrical Engineering, Faculty of Engineering, University of Kufa, Iraq \\ ${ }^{1,2}$ Wireless and Radio Science Centre (WARAS), Faculty of Electrical and Electronic Engineering, \\ Universiti Tun Hussein Onn Malaysia, Malaysia
}

\begin{tabular}{l} 
Article Info \\
\hline Article histo \\
Received A \\
Revised Jun \\
Accepted Ju \\
\hline Keywords: \\
5G \\
BCH \\
f-OFDM \\
OOBE \\
PAPR
\end{tabular}

\section{Article Info}

icle history:

Received Apr 5, 2019

Revised Jun 6, 2019

In modern society, the mobile communications are playing an important role. Since 1980's, the first generation $(1 \mathrm{G})$ was appeared which was providing up to $2.4 \mathrm{Kbps}$ data rate. Whilst in the last of 1980's, second generation $(2 \mathrm{G})$ has been began used rather than $1 \mathrm{G}$ because of it is some limitations in $1 \mathrm{G}$. It was providing up to $64 \mathrm{Kbps}$ data rate and then arrived to around $100 \mathrm{Kbps}$ through its upgraded. Whereas in 2000 's, third generations has been appeared to provide up to $2 \mathrm{Mbps}$ data rate. It was characterized of high speed mobile access, mobile television and video calling. In contrast, Third Generation Partnership Project (3GPP) could providing $2 \mathrm{Mbps}$ data rate in low mobility and from $144-384 \mathrm{Kbps}$ in high mobility for $2100 \mathrm{MHz}$ band frequency. Fourth Generation (4G) which is providing higher data rates is characterized of Digital video chat, mobile TV, Video Broadcasting (DVB) and high definition TV content. Orthogonal

\begin{abstract}
Due to its large peak to average power ratio (PAPR) and high out of band Additionally, it bandwidth. In contrast, f-OFDM is dividing the system's bandwidth into a number of subbands to support several waveform parameters based on various service scenarios. So, Filtered-OFDM (f-OFDM) is considered as a modern enabler of the flexible waveform to overcome the OFDM drawbacks while remaining its advantages as well as, to encounter the new challenges that faced 5G. Nonetheless, there is a trade-off among OOBE, PAPR and SNR performance. Meanwhile, channel coding technology is one of the most important issue in physical layer which is playing an essential role in order to achieve the reliability and latency. So, BCH code has been suggested in this paper for f-OFDM system to achieve the reliability of transmission information and thus improving BER performance over multipath fading channel. Whilst, BCH-LTE system is introduced as a baseline in this paper that using for comparison purpose with proposed system. Simulation results showed that the proposed BCH-f-OFDM system was significantly better than BCH-LTE system in terms of decreasing OOBE and achieving improving in BER performance. Although, PAPR levels was stilling high in proposed system due to the trade-off among OOBE, PAPR and SNR performance. However, the proposed system is considered a promising candidate to meet the requirements of $5 \mathrm{G}$ services because of its ability to solve two important
\end{abstract}

Copyright $@ 2020$ Institute of Advanced Engineering and Science. All rights reserved.
Ghasan Ali Hussain,

Department of Electrical Engineering,

Faculty of Engineering,

University of Kufa, Iraq.

Email: ghasan.alabaichy@uokufa.edu.iq 
Frequency Division Multiplexing (OFDM) and Multi Input Multi Output (MIMO) antennas technologies are used in 4G. Now, all interests heading towards the emergence of the fifth generation which is characterized of high capacity, low latency, higher data rate and massive device connectivity through combining different techniques including of adaptive modulation schemes and advanced multiple antenna techniques. The major objectives for some of mobile communication are needing to improve in near future due to the needing of increase the capacity, improve the quality of service, lower latency and higher data rate. Thus, the modification at physical layer should be done in terms of waveforms [1].

Although, OFDM can improve spectrum efficiency and by using cyclic prefix can resist both of inter-symbol interference (ISI) and inter-carrier interference (ICI) in previous systems [2]. However, there are some of limitations with $5 \mathrm{G}$, leading to a consideration of another waveform which are gives a solution for these limitations. The reason behind the trend of using multicarrier modulation is because of its simple implementation and easier equalization specially with higher bandwidth [1].

Because of its PAPR and large OOBE, OFDM doesn't meet the requirements of 5G services. Additionally, it supports only one type of waveform parameters in entire bandwidth. Whilst, f-OFDM that created by modify OFDM is dividing the system's bandwidth into a number of subbands, each subband then filtered via various filter and thus support several waveform parameters based on various service scenarios [3].

In the past few years, several studies have been discussed different candidate waveforms of $5 \mathrm{G}$ such as Filter Bank Multi carrier modulation (FBMC) in [4] which has been compared with OFDM technique for 5G. The comparison included the power spectral density, prototype filter and computational complexity. They showed that, although FBMC overcome the limitations of OFDM in terms of conserves the bandwidth and lower side lobes unlike OFDM that have high and overlapping side lobes additionally it sacrifices the bandwidth that required by its cyclic prefix. However, the FBMC demerits are high computation complexity and limited flexibility unlike OFDM that characterized of lower complexity as well as higher flexibility especially with MIMO techniques [4-7].

Universal Filtered Multi Carrier (UFMC) which generalization of FBMC and f-OFDM has been proposed as promising waveform contender for 5G in [8]. They revealed that the values of PAPR for UFMC is better than OFDM with the exception of 4QAM mapper method. Although, the performance of UFMC is better than OFDM in terms of BER for discrete narrow band networks and side lobe attenuation. However, extra filtering need to increase the implementation complexity [8]. In other word, both of FBMC and UFMC are characterized of lower OOBE than OFDM, but their demerits are shown in implementation complexity as well as their performance that degraded with MIMO channels unlike OFDM [9].

In contrast, Generalized Frequency Division Multiplexing (GFDM) has been proposed for future 5G networks in [10] as a contender waveform for air interface. The error rate performance has been discussed for GFDM under different channel conditions. They explained that the possibility of considering GFDM as a novel modulation technology which satisfy the next generation requirements of mobile wireless systems. However, there are several issues that didn't solved [10]. Where, GFDM needs to tail biting and high-order filtering to suppress inter-subcarrier interference due to its subcarriers are not mutually orthogonal and arranged in close proximity, unlike f-OFDM that have subcarriers in each subband that still quasi-orthogonal and it has relatively short filter length as well as no need to complicated processing [11].

Meanwhile, f-OFDM has been presented in [11] for 5G as modern enabler of the flexible waveform to avoid the limitations of OFDM and to encounter the new challenges that faced 5G. Filtered-OFDM could overcoming of the OFDM drawbacks while remaining its advantages. Where, by suitable design of f-OFDM filters, the OOBE can be suppressed and reducing the consumption of guard band. Furthermore, could applying optimized numerology through each subband to fit the needs of particular kind of services. Thus, considering f-OFDM as a most promising waveform can be fulfilling the $5 \mathrm{G}$ requirements and the whole spectrum efficiency [11]. Moreover, because of a low complexity and high multiplexing flexibility preserving same of OFDM, f-OFDM is considered one of the most promising and competitor waveform for $5 \mathrm{G}$ comparing to other contender waveforms such as GFDM, FBMC and UFMC. Where, f-OFDM can achieve forward and backward compatibility, low OOBE, reasonable complexity and comfortable synchronization through a suitable filter design [12].

Channel coding technology is considered one of the most important issue in physical layer which is playing an important role in order to achieve the reliability and latency [13]. Where, different kinds of channel coding including of turbo, LDPC, convolutional, $\mathrm{BCH}$ and RS have been proposed to achieve the reliability in previous LTE systems [14-18]. Whereas, channel coding such as turbo, polar, LDPC and convolutional codes have been also proposed for $5 \mathrm{G}$ in [13]. They concluded that considerable improvements in the performance could be achieved in short message length regime in the next generation when exchanging other modern coding schemes instead of LTE-Turbo code at the expense of increasing decoder complexity. In contrast, two new channel coding (polar and LDPC) codes are used in data and control channel of 5G new 
radio (NR) air interface in [19]. They showed the capability of NR LDPC of achieving lower latency and higher throughput than LTE turbo codes as well as NR polar codes outperform of LTE TBCCs. Nevertheless, the NR polar code complexity is considerably higher than LTE TBCCs. On the other hand, both [20, 21] are proposed polar codes for $5 \mathrm{G}$ and compared their performance with LTE turbo codes. They concluded that the considerably performance gain achieved by polar codes over turbo codes.

In noisy channels, using error correction code (ECC) is considered an important for digital communication systems to achieve the reliability of the transmission information [22]. In practical applications, Bose-Chaudhuri- Hocquenghem $(\mathrm{BCH})$ is usually adopted to achieve data reliability among the several ECCs because of its acceptable hardware cost and strong error correcting performance [23]. Furthermore, $\mathrm{BCH}$ code outperforms both of turbo and convolutional codes with LTE system in terms of BER performance and lower complexity [17].

To the best of our knowledge there are no proposing $\mathrm{BCH}$ code yet in f-OFDM system that considered as contender candidate waveform of $5 \mathrm{G}$. So, $\mathrm{BCH}$ codes have been proposed in this paper for $\mathrm{f}$ OFDM to achieve reliable data transmission. BCH-LTE system [17] is introduced in this paper as baseline that using for comparison purpose with the proposed system. The comparison will be including all of BER performance, OOBE and PAPR levels.

The rest of paper will be consisting of following: Section 2, the proposed system that described the f-OFDM system and all parameters, Section 3, including the results and discussion. The conclusion will be presented in the last section.

\section{PROPOSED SYSTEM}

Based on BCH-LTE system [17] which is introduced as baseline in this paper. The block diagram of proposed system by using $\mathrm{BCH}$ code in f-OFMD has been shown in Figure 1. Where, $\mathrm{BCH}(15,5)$ codes has been used with f-OFDM system rather than OFDM to be offered as strong contender waveform of 5G that solving demerits of OFDM with maintaining of its advantages.

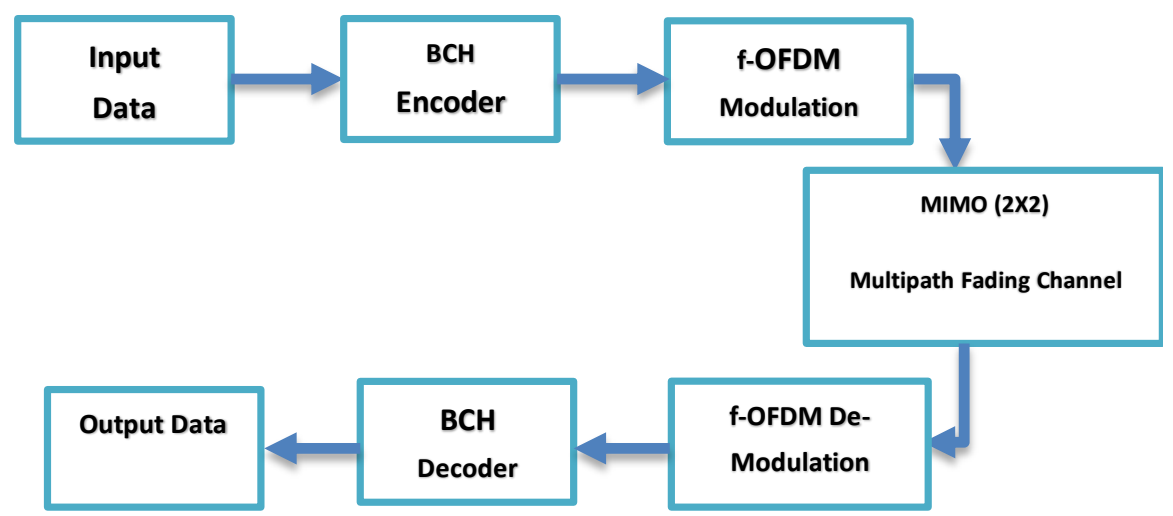

Figure 1. BCH code for Filtered-OFDM system

Table 1. Simulation Parameters

\begin{tabular}{cc}
\hline Transmission Bandwidth & $20 \mathrm{MHz}$ \\
\hline Channel & 2X2 MIMO Channel \\
& Multipath Fading \\
& Channel \\
Number of IFFT/FFT Points & 2048 \\
No. of Occupied Sub-carrier & 1200 \\
Cyclic Prefix Length & 144 \\
Modulation & BPSK \\
Subcarrier Spacing & $15 \mathrm{KHz}$ \\
& \\
Bandwidth & $19.83 \mathrm{MHz}$ \\
Sampling rate & $30.72 \mathrm{MHz}$ \\
Type & RRC Windowed- \\
Filter & Sinc \\
Design & 0.6 \\
Roll-off factor $(\alpha)$ & 513 \\
Length & BCH (15,5) Codes \\
\hline
\end{tabular}

Indonesian J Elec Eng \& Comp Sci, Vol. 17, No. 1, January 2020 : 310-316 
The parameters that applied in this paper has been shown in Table 1. Where the same parameters of the baseline BCH-LTE system are used in order to compare both systems under the same parameters. Additionally, 2X2 MIMO multipath fading channel of baseline BCH-LTE system has been also chosen here to transmit and receive the signal to simulate the system under multipath fading channel. In contrast, BPSK modulation scheme has been used in this paper due to the lower modulation schemes are improving the system performance better than higher modulation schemes [24].

The filter in f-OFDM system has been designed in this paper depending on FIR digital filter based on window function method in order achieving very low OOBE. Where, by using window function will be obtaining lower OOBE performance through occupying a bigger filter's bandwidth [3]. The idea in f-OFDM is representing by recusing the interferences between neighbouring subbands, where each subband of the baseband OFDM signals is filtered via a band-limited filter to combat its OOBE, thus minimizing the interference from neighbouring subbanns to enough level [25].

\section{RESULTS AND DISCUSSION}

The Proposed f-OFDM system by using BCH code has been simulated by MATLAB over (2X2) MIMO multipath fading channel. Three issues in f-OFDM have been discussed and compare their results with OFDM system to show the beneficial of proposing f-OFDM rather than OFDM for 5G. In other hand, different techniques have been proposed to decrease OOBE, however they faced the challenge of trade-off among OOBE, PPAR and SNR performance [26]. Therefore, achieve balanced between minimize OOBE level and obtaining of accepted values for both PAPR and SNR performance is considered very important issues. First, the OOBE level will be discussed and showing in Figure 2.
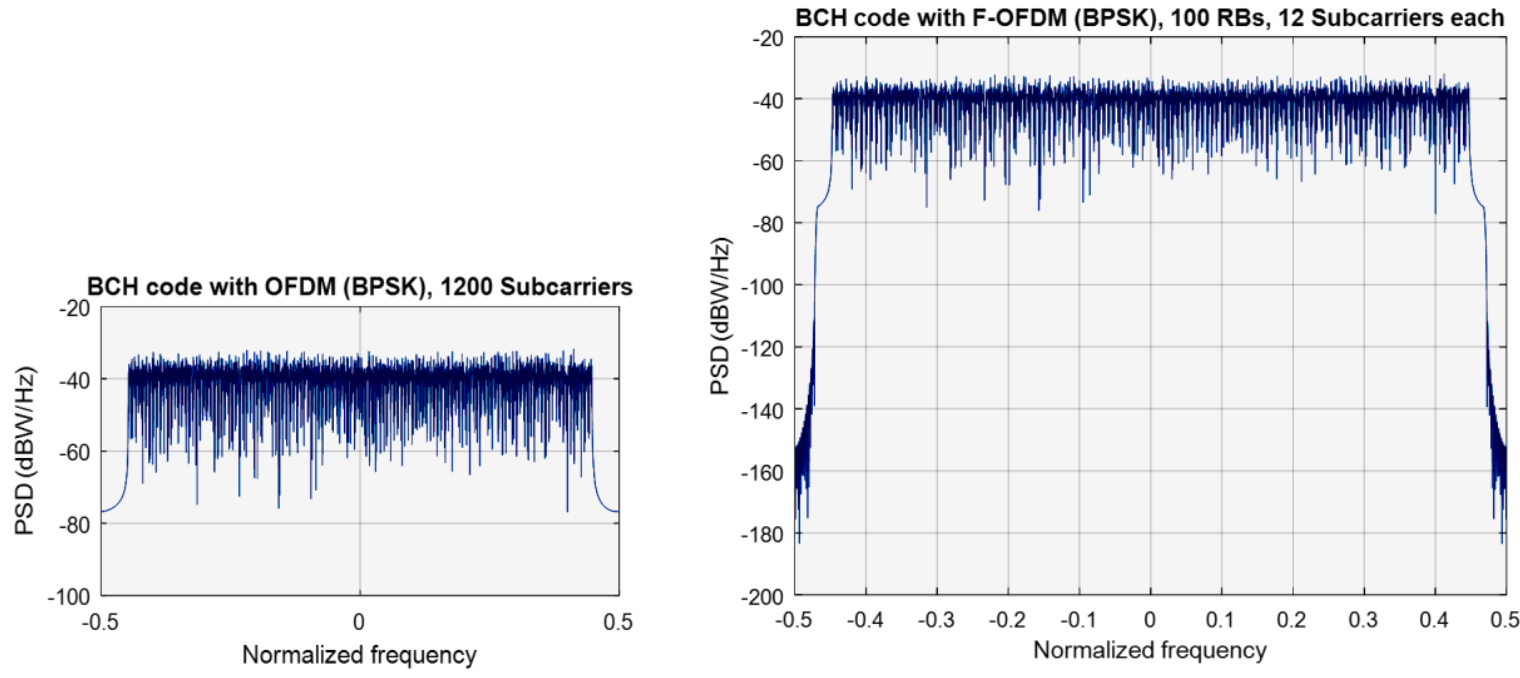

Figure 2. Power Spectral Density of OFDM system versus proposed f-OFDM system/BPSK

As shown in Figure 2, power spectral density (PSD) of OFDM system is very high comparing with f-OFDM system. Where, it was around $-80 \mathrm{~dB}$ in OFDM and $-180 \mathrm{~dB}$ in proposed f-OFDM system. Thus the proposed $\mathrm{f}-\mathrm{OFDM}$ system could be achieving around 100dB lower than conventional OFDM system with BPSK modulation scheme. Therefore, by proposing f-OFDM system could resolving the first problem of OFDM system.

While, the second issue is BER performance of f-OFDM system and compared with OFDM system. The purpose of discussed this issue is to show the impact of decreasing OOBE on the system performance and if that will be degrading the BER performance or not. The BER performance of f-OFDM system versus OFDM system has been shown in Figure 3.

As shown in Figure 3, the performance of uncoded f-OFDM system was somehow close to the performance of uncoded OFDM and then both of systems are clearly improved when using BCH codes. While, the improvements in f-OFDM system was more than OFDM system. So, proposing BCH codes in fOFDM is contributes significantly in enhancing system performance better than in OFDM. Thus, by using $\mathrm{BCH}$ codes in $\mathrm{f}-\mathrm{OFDM}$ could able to solve the second challenge. 


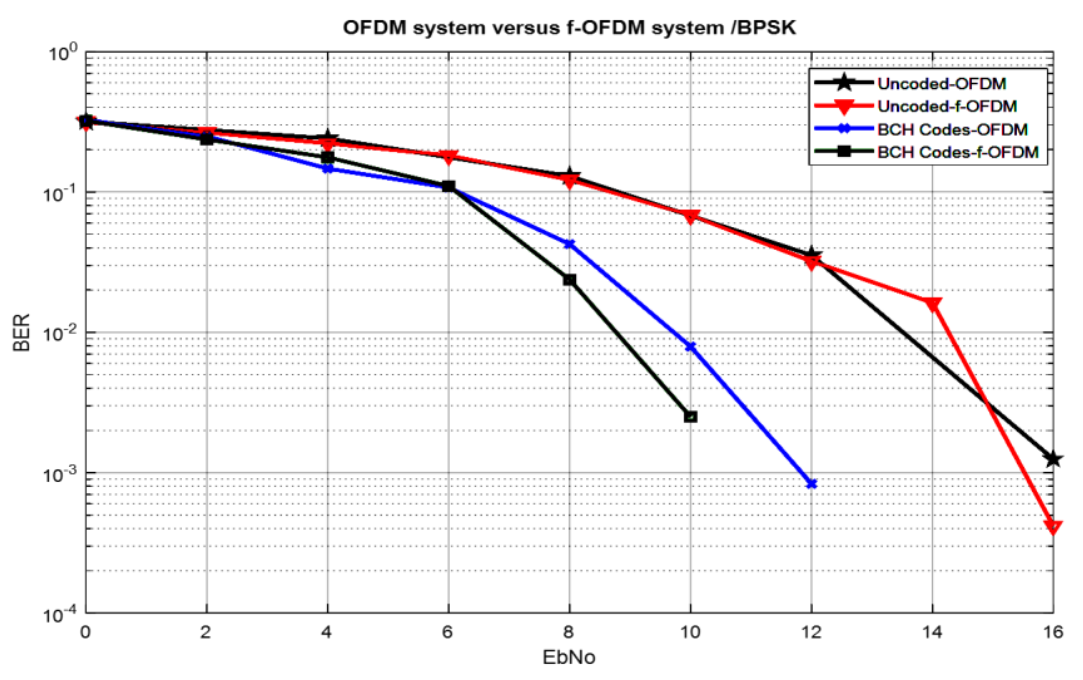

Figure 3. BER performance of proposed Filtered-OFDM system versus OFDM system/ BPSK

Third issue is the PAPR level of f-OFDM system and compared with OFDM system. Table 2, shows the level of PAPR for both of f-OFDM and OFDM systems. As shown in Table 2, the PAPR level of fOFDM system was higher than OFDM system. Although, the proposed f-OFDM system has achieved good results of OOBE and BER performance compared to OFDM system but it couldn't reduce the PAPR level of its system. Thus, it needs to other techniques or modifications to reduce the PAPR with taking in the account maintain both improvements of BER and reducing OOB.

Nevertheless, the proposed f-OFDM system performance is still considered a good candidate waveform for $5 \mathrm{G}$ because of it is solving the disadvantages of OFDM system such as high OOBE, furthermore the performance of f-OFDM system could be improving better than OFDM when using $\mathrm{BCH}$ codes.

Table 2. PAPR OF OFDM Versus f-OFDM

\begin{tabular}{cc}
\hline Technique & PAPR \\
\hline OFDM & $9.5235 \mathrm{~dB}$ \\
f-OFDM & $9.7868 \mathrm{~dB}$ \\
\hline
\end{tabular}

\section{CONCLUSION}

In this paper, $\mathrm{BCH}$ code has been suggested in $\mathrm{f}-\mathrm{OFDM}$ system to achieve the reliability via multipath fading channel to presented as a one of the most contender waveforms for 5G. Here, FilteredOFDM has been proposed to be used rather than OFDM for next generation of mobile communications, due to its capability of overcoming the OFDM limitation with maintains of its advantages. Different issues have been discussed in this paper to show the advantages of proposing f-OFDM system in 5G. The results reveal large improvements in terms of the OOBE reduction, where the proposed f-OFDM system could be minimizing around $100 \mathrm{~dB}$ lower than conventional OFDM system. In the other hand, the improvements of BER performance by proposing $\mathrm{BCH}$ codes in $\mathrm{f}-\mathrm{OFDM}$ system was significantly better than in OFDM system. Thus, the proposed f-OFDM system is succeeded of reducing OOBE as well as improved its system performance by using $\mathrm{BCH}$ codes better than OFDM system performance. Where, using BCH codes helped in the correcting the errors in the transmission channel in f-OFDM, and thus enhanced its system performance. Although, the proposed f-OFDM system couldn't reduce the PAPR level because of the tradeoffs among OOBE, PPAR and SNR performance. However, the proposed system could be achieving very low OOBE and enhancing BER performance better than OFDM system when using BCH codes, so the proposed system is presented as a one of the most contender waveform to fulfilment the requirements of $5 \mathrm{G}$.

Due to couldn't reducing PAPR levels in this paper which is considered as limitation of BCH-fOFDM system here. Looking for modifications or new techniques that have the capability of reducing PAPR in f-OFDM system while maintains of its low OOBE levels and the achieved BER performances is recommended to be as interesting future work in this field. 


\section{ACKNOWLEDGEMENTS}

This research was funded by the Ministry of Higher Education Malaysia under Fundamental Research Grant Scheme Vot No. 1627 and partially sponsored by Universiti Tun Hussein Onn Malaysia.

\section{REFERENCES}

[1] H. Jebbar, S. El Hassani, A. El Abbassi," Performance study of 5G multicarrier waveforms", 2017 International Conference on Wireless Networks and Mobile Communications (WINCOM), Nov. 2017.

[2] A. Hammoodi, L. Audah, M. A. Taher, " Green Coexistence for 5G Waveform Candidates: A Review", IEEE Access, vol. 7, pp. 1-24, 2019.

[3] X. Cheng, Y. He, B. Ge, C. He," A Filtered OFDM Using FIR Filter Based on Window Function Method", 2016 IEEE 83rd Vehicular Technology Conference (VTC Spring), 2016.

[4] P. Kansal, A. K. Shankhwar, " FBMC vs OFDM Waveform Contenders for 5G Wireless Communication System", Wireless Engineering and Technology, vol. 8, no. 9, pp. 59-70, Oct. 2017.

[5] B. F. Boroujrny, " OFDM Versus Filter Bank Multicarrier", IEEE Signal Processing Magazine, vol. 28, no. 3, pp. 92-112, May 2001.

[6] J. Li, E. Bala, R. Yang, " Resource block Filtered-OFDM for future spectrally agile and power efficient systems", ScienceDirect, ELSEVIER, Physical Communication, vol. 11, pp. 36-55, June, 2014.

[7] L. Zhang, A. Ijaz, P. Xiao, R. Tafazolli, " Multi-Service System: An Enabler of Flexible 5G Air Interface," IEEE Communications Magazine, vol. 55, no. 10, pp. 152-159, Oct. 2017.

[8] P. N. Rani, Ch. S. Rani," UFMC: The 5G modulation technique", 2016 IEEE International Conference on Computational Intelligence and Computing Research (ICCIC), Dec. 2016.

[9] M. Mohamad, R. Nilsson, J. v. de Beek, " An analysis of out-of-band emission and in-band interference for precoded and classical OFDM systems", Proceedings of European Wireless 2015; 21th European Wireless Conference, May 2015.

[10] N. Michailow, M. Matthé, I. S. Gaspar, A. N. Caldevilla, L. L. Mend, A. Festag, "Generalized Frequency Division Multiplexing for 5th Generation Cellular Sep. 2014.Networks," IEEE Transactions on Communications, vol. 62 , no. 9, pp. 3045-3061, Sep. 2014.

[11] X. Zhang, M. Jia, L. Chen, J. Ma, J. Qiu," Filtered-OFDM - Enabler for Flexible Waveform in the 5th Generation Cellular Networks", 2015 IEEE Global Communications Conference (GLOBECOM), Dec. 2015.

[12] J. Wang, A. Jin, D. Shi, L. Wang, H. Shen, D. Wu, L. Hu, L. Gu, L. Lu, Y. Chen, J. Wang, Y. Saito, A. Benjebbour, Y. Kishiyama," Spectral Efficiency Improvement with 5G Technologies: Results From Field Tests", IEEE Journal on Selected Areas in Communications, vol. 35, no. 8, pp. 1867-1875, June 2017.

[13] O. Iscan, D. Lentner, W. Xu," A Comparison of Channel Coding Schemes for $5 G$ Short Message Transmission", 2016 IEEE Globecom Workshops (GC Wkshps), Dec. 2016.

[14] A. Li, L. Xiang, T. Chen, R. G. Maunder, B. M. Al-Hashimi, L. Hanzo," VLSI Implementation of Fully Parallel LTE Turbo Decoders", IEEE Access, vol. 4, pp. 323-346, Jan. 2016.

[15] I. Bhurtah, P. C. Catherine, K M S. Soyjaudah, "Enhancing the Error-Correcting Performance of LDPC Codes for LTE and WiFi", International Conference on Computing, Communication \& Automation, May 2015.

[16] L. Li-fu, L. Hai-wen, L. Hong-liang, G. Yong-jun," Research and implementation of Viterbi decoding in TD-LTE system", 2017 IEEE 2nd Advanced Information Technology, Electronic and Automation Control Conference (IAEAC), March 2017.

[17] G. A. Hussain, L. Audah," Downlink LTE System Performance Improvement by Using BCH Codes over LTEMIMO Channel", International Journal of Integrated Engineering, vol. 10, no. 4, pp. 95-101, 2018.

[18] G. A. Hussain, L. Audah," RS Codes Downlink LTE System over LTE-MIMO Channel", TELKOMNIKA Telecommunication, Computing, Electronics and Control, vol. 16, no. 6, pp. 281-286, 2018.

[19] D. Hui, S. Sandberg, Y. Blankenship, M. Andersson, L. Grosjean," Channel Coding in 5G New Radio: A Tutorial Overview and Performance Comparison with 4G LTE", IEEE Vehicular Technology Magazine, vol. 13, no. 4, Oct. 2018.

[20] B. Zhang, H. Shen, B. Yin, L. Lu, D. Chen, T. Wang, L. Gu, X. Wang, X. Hou, H. Jiang, A. Benjebbour, Y. Kishiyama," A 5G Trial of Polar Code", 2016 IEEE Globecom Workshops (GC Wkshps), Dec. 2016.

[21] P. Chen, M. Xu, B. Bai, J. Wang, " Design and Performance of Polar Codes for 5G Communication under High Mobility Scenarios", 2017 IEEE 85th Vehicular Technology Conference (VTC Spring), June 2017.

[22] D. Jo, S. Kwon, D. Shin, "Blind Reconstruction of BCH Codes Based on Consecutive Roots of Generator Polynomials", IEEE Communications Letters, vol. 22, no. 5, pp. 894-897, May 2018.

[23] B. Park, J. Park, Y. Lee," Area-Optimized Fully-Flexible BCH Decoder for Multiple GF Dimensions", IEEE Access, vol. 6, pp. 14498-14509, March 2018.

[24] M. A. N. Sukar, M. Pal," SC-FDMA \& OFDMA in LTE physical layer", International Journal of Engineering Trends and Technology (IJETT), vol. 12, no. 2, pp. 74-85, Jun 2014.

[25] D. Wu, X. Zhang, J. Qiu, L. Gu, Y. Saito, A. Benjebbour, Y. Kishiyama, " A Field Trial of f-OFDM toward 5G," 2016 IEEE Globecom Workshops (GC Wkshps), Dec. 2016.

[26] A. Selim, L. Doyle," A method for reducing the out-of-band emissions for OFDM systems," 2014 IEEE Wireless Communications and Networking Conference (WCNC), April 2014. 


\section{BIOGRAPHIES OF AUTHORS}

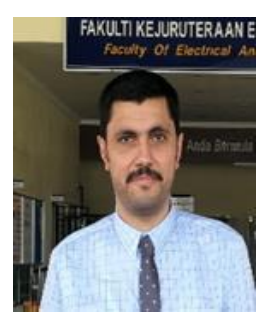

Ghasan A. Hussain* is currently a PhD student in Universiti Tun Hussein Onn Malaysia since 2017. He received Bachelor degree in Communication Engineering from Alfatah UniversityTripoli/Libya in 2003. Hussian received the Master degree in communication Engineering from University Putra Malaysia in 2013. Since 2006, he has been with Electrical Engineering, University of Kufa, Iraq, where he is now a lecturer. His research interests include wireless communication systems, Mobile systems, LTE and Error Correction Techniques. He has a number of publications in different journals in his field.

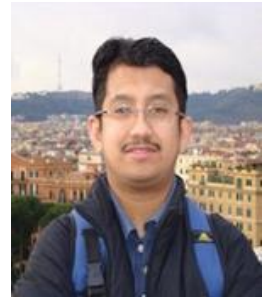

Lukman Audah received his BS degree in electrical engineering from the University Technology Malaysia, Johor, Malaysia, in 2005, and his MS and PhD degrees in communication networks and software from the University of Surrey, United Kingdom, in 2007 and 2013, respectively. Since 2014, he has been with the Faculty of Electrical and Electronic Engineering, University Tun Hussein Onn Malaysia, Johor, Malaysia, where he is now a Senior lecturer. His main research interests are wireless and mobile communications, Internet traffic engineering, network system management, data security, and satellite communications. 